\title{
Coronal mass ejections, type II radio bursts, and solar energetic particle events in the SOHO era
}

\author{
N. Gopalswamy ${ }^{1}$, S. Yashiro ${ }^{2}$, S. Akiyama ${ }^{2}$, P. Mäkelä ${ }^{2}$, H. Xie ${ }^{2}$, M. L. Kaiser ${ }^{1}$, R. A. Howard ${ }^{3}$, and J. L. Bougeret ${ }^{4}$ \\ ${ }^{1}$ NASA Goddard Space Flight Center, Greenbelt, MD, USA \\ ${ }^{2}$ The Catholic University of America, Washington D.C., USA \\ ${ }^{3}$ Naval Research Laboratory, Washington D.C., USA \\ ${ }^{4}$ Paris Observatory, Meudon, France
}

Received: 8 October 2007 - Revised: 13 February 2008 - Accepted: 13 February 2008 - Published: 15 October 2008

\begin{abstract}
Using the extensive and uniform data on coronal mass ejections (CMEs), solar energetic particle (SEP) events, and type II radio bursts during the $\mathrm{SOHO}$ era, we discuss how the CME properties such as speed, width and solar-source longitude decide whether CMEs are associated with type II radio bursts and SEP events. We discuss why some radio-quiet CMEs are associated with small SEP events while some radio-loud CMEs are not associated with SEP events. We conclude that either some fast and wide CMEs do not drive shocks or they drive weak shocks that do not produce significant levels of particle acceleration. We also infer that the Alfvén speed in the corona and near-Sun interplanetary medium ranges from $<200 \mathrm{~km} / \mathrm{s}$ to $\sim 1600 \mathrm{~km} / \mathrm{s}$. Radio-quiet fast and wide CMEs are also poor SEP producers and the association rate of type II bursts and SEP events steadily increases with CME speed and width (i.e. energy). If we consider western hemispheric CMEs, the SEP association rate increases linearly from $\sim 30 \%$ for $800 \mathrm{~km} / \mathrm{s}$ CMEs to $100 \%$ for $\geq 1800 \mathrm{~km} / \mathrm{s}$. Essentially all type II bursts in the decametre-hectometric (DH) wavelength range are associated with SEP events once the source location on the Sun is taken into account. This is a significant result for space weather applications, because if a CME originating from the western hemisphere is accompanied by a DH type II burst, there is a high probability that it will produce an SEP event.
\end{abstract}

Keywords. Interplanetary physics (Interplanetary shocks) Solar physics, astrophysics, and astronomy (Energetic particles; Flares and mass ejections; Radio emissions)

Correspondence to: N. Gopalswamy

(nat.gopalswamy@nasa.gov)

\section{Introduction}

Coronal mass ejections (CMEs) associated with type radio II bursts are more energetic on the average and there is a hierarchical relationship between CME kinetic energy and the wavelength range of type II radio bursts (Gopalswamy et al., 2001a, 2005; Lara et al., 2003; Gopalswamy, 2006). The average speeds of CMEs associated with type II bursts confined to metric wavelengths is $610 \mathrm{~km} / \mathrm{s}$, only about $30 \%$ higher than the average speed $(\sim 470 \mathrm{~km} / \mathrm{s})$ of all CMEs. On the other hand type II bursts with emission in the metric to kilometric wavelengths (the so-called mkm type II bursts) are associated with CMEs of higher average speed: $1490 \mathrm{~km} / \mathrm{s}$, which is $\sim 3$ times the average speed of all CMEs. CMEs associated with decameter-hectometric (DH) type II bursts have intermediate speed: $1115 \mathrm{~km} / \mathrm{s}$. Interestingly, the average speed $(1524 \mathrm{~km} / \mathrm{s})$ of CMEs associated with large solar energetic particle (SEP) events is very similar to that of CMEs with mkm type II bursts. This is consistent with the idea that CME-driven shocks accelerate both ions and electrons. Statistical studies have confirmed this close association (see e.g. Gopalswamy, 2003; Cliver et al., 2004a). Thus, type II bursts, especially those occurring at longer wavelengths, have become good indicators of SEP events.

There are some observations that contradict the above picture. It was recognized long ago that some fast CMEs observed during 1979-1982 were not associated with metric type II bursts (Sheeley et al., 1984). These CMEs had speeds up to $1600 \mathrm{~km} / \mathrm{s}$ with a median value of $\sim 455 \mathrm{~km} / \mathrm{s}$. When CMEs move faster than the characteristic speed of the ambient medium (say, the Alfvén speed), they drive fast-mode MHD shocks, which in turn accelerate electrons to produce the type II bursts. In this scheme, the radio-quietness (i.e. the lack of type II bursts in the metric and DH wavelengths) can be explained as being due to either the fast CMEs not

Published by Copernicus Publications on behalf of the European Geosciences Union. 
attaining super-Alfvénic speeds, or the CME-driven shocks are unable to excite type II emission (Sheeley et al., 1984). Gopalswamy et al. (2001b) found that about a third of CMEs faster than $\sim 900 \mathrm{~km} / \mathrm{s}$ were not associated with DH type II bursts observed by the WAVES experiment (Bougeret et al., 1995 ) on board the Wind spacecraft. Recently Gopalswamy et al. (2008) found even a larger fraction $(\sim 42 \%)$ of fast (speed $\geq 900 \mathrm{~km} / \mathrm{s}$ ) and wide (width $\geq 60^{\circ}$ ) CMEs were radio quiet (RQ). The width criterion selects more energetic CMEs because the CME width (W) is statistically related to the mass (M) by the relation, $\log \mathrm{M}=12.6+1.3 \log \mathrm{W}$ (Gopalswamy et al., 2005b). This relation translates the width criterion into a mass criterion, so $\mathrm{W}=60^{\circ}$ corresponds to a mass of $8.2 \times 10^{14} \mathrm{~g}$. The corresponding CME kinetic energy is $\sim 3.3 \times 10^{30} \mathrm{erg}$, which is slightly above the average kinetic energy of all CMEs (Gopalswamy, 2004). Radio quietness even at such energies suggests that these high-speed CMEs may not be super-Alfvénic. One of the reasons for studying the type II burst association with CMEs is that they are indicative of particle acceleration (electrons and ions) by CME-driven shocks. Not all radio-loud (RL) CMEs are associated with SEP events, so we do not know how reliable are type II bursts as SEP indicators. Thus to get a complete picture on the ability of CMEs in driving shocks and producing energetic particles, we need to understand how CMEs, SEPs, and type II radio bursts are interrelated. We consider these issues using the extensive database on CMEs, SEPs, type II radio bursts which covers almost the entire solar cycle 23 .

In Sect. 2, we consider the properties of RQ and RL fast and wide (FW) CMEs. We also compare the properties all CMEs associated with DH type II bursts (irrespective of the CME speed) with those of FW CMEs. In Sect. 3, we consider the rate at which DH type II bursts are associated CMEs as a function of CME speed, width, and source longitude for FW CMEs and all CMEs. In Sect. 4, we investigate the association of SEP events with RQ and RL CMEs. In particular we examine why some RQ CMEs are associated with SEP events and why some RL CMEs are not associated with SEP events. In Sect. 5, we discuss the influence of the ambient medium on the shock-driving ability of CMEs. We present our summary and conclusions in Sect. 6 .

\section{CMEs with and without type II radio bursts}

Applying the speed and width criteria (speed $\geq 900 \mathrm{~km} / \mathrm{s}$ and width $\geq 60^{\circ}$ ), $472 \mathrm{FW}$ CMEs have been identified during 1996 to 2005 (Gopalswamy et al., 2008). The FW CMEs constitute just $5 \%$ of all CMEs ( $>10000)$ recorded by the Solar and Heliospheric Observatory (SOHO) mission's Large Angle and Spectrometric Coronagraph (LASCO, Brueckner et al., 1995) over the same period (Yashiro et al., 2004). The 472 FW CMEs fall into 193 RQ and 268 RL CMEs. For 11 FW CMEs, there are no radio data. In addition to these RL (FW) CMEs, we consider here another population of CMEs associated with DH type II bursts, irrespective of the existence of metric or kilometric type II bursts; we refer to this population as RL (DH) CMEs, which can have speeds less than $900 \mathrm{~km} / \mathrm{s}$. Note that our RQ and RL (FW) CMEs form the two complementary subsets of FW CMEs. The RL (DH) CMEs are not a subset of FW CMEs.

To check whether an FW CME is RQ or RL, we look for type II burst association in the metric and DH wavelengths. Our experience is that metric and DH type II bursts are delayed by about $20 \mathrm{~min}$ and $25 \mathrm{~min}$, respectively, from the CME onset. When there is a type II burst in the window 30 min before and 60 min after the CME onset, we term the CME RL, otherwise RQ. If there is a type II burst beyond this window, we consider this as an association after carefully ensuring that the burst could not be due to a different CME. To check the CME association of DH type II bursts, we looked for CME onset preceding the type II generally within an hour. The time difference is occasionally longer for type II bursts starting at very low frequencies $(\sim 1 \mathrm{MHz})$. Information on metric type II bursts is obtained from the Solar Geophysical Data archived at the National Geophysical Data Center (ftp://ftp.ngdc.noaa.gov/STP/SOLAR_DATA/ SOLAR_RADIO/SPECTRAL/Type_II_1994-2007). Information on DH type II bursts is also available online as a preliminary catalog (http://lep694.gsfc.nasa.gov/waves/ waves.html). A list of DH type II bursts with information on the associated CMEs is also available online at the CDAW Data Center (http://cdaw.gsfc.nasa.gov/CME_ list/radio/waves_type2.html).

\subsection{Annual number of FW CMEs}

It is instructive to consider how the number of FW CMEs varies with time. Figure 1 shows that the number of FW CMEs varies by an order of magnitude between the minimum and maximum phases of solar cycle 23 . This is also true for the RL and RQ subsets. The year 2002 has the largest number, which is the year of the second maximum in the CME rate (Gopalswamy, 2004). During each year, the ordering of CME numbers among the three populations is similar: \#FW $>\# R L \geq \# R Q$, except for the year 1999 when $\# R L<\# R Q$. The peculiar behaviour in the year 1999 has been noticed in other energetic CME populations as well: the number of geoeffective halo CMEs (Gopalswamy et al., 2007), and the number of SEPeffective CMEs (Gopalswamy et al., 2003). The selection criterion requires that the CMEs be faster than $900 \mathrm{~km} / \mathrm{s}$, so the annual number of FW CMEs generally follows the speed distribution (Gopalswamy et al., 2006) shown in Fig. 1.

\subsection{Kinematic properties of FW CMEs}

Table 1 summarizes the properties of the RL (FW) and RQ CMEs extracted from Gopalswamy et al. (2008). The RQ CMEs are slower, have less number of halos, are associated 

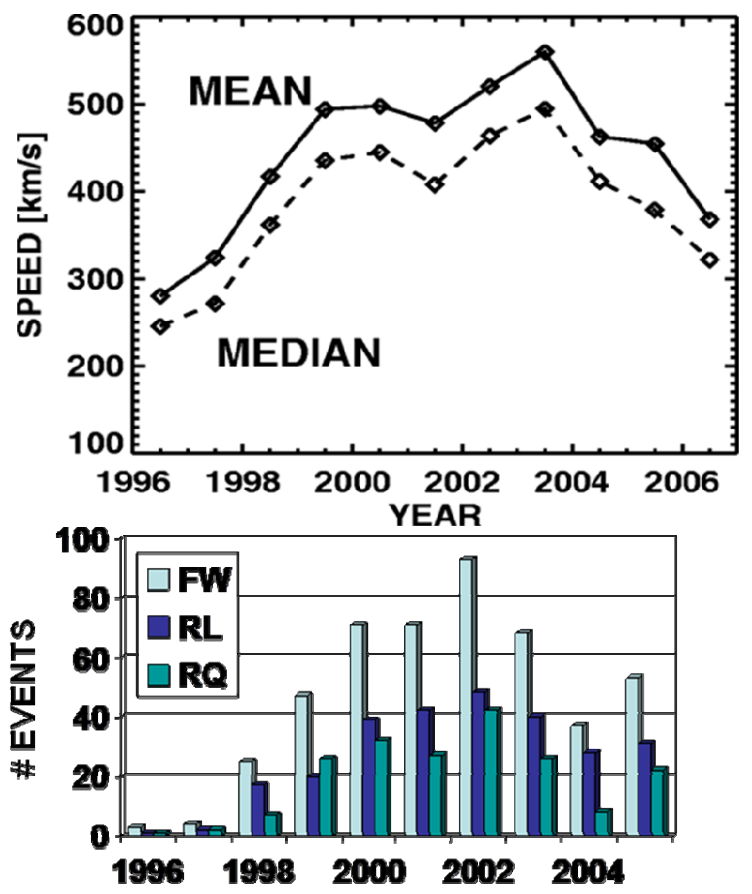

Fig. 1. Annual numbers of FW CMEs and the RQ and RL subsets for the years 1996 to 2005 (bottom). The annual variation of the mean CME speed (top) is also shown for comparison.

with weaker flares, and are ejected at large angles to the Sun-Earth line. No large SEP events are associated with RQ CMEs, while about half of the RL CMEs are. Large or major SEP events are those with proton intensity (Ip) at least $10 \mathrm{pfu}$ [particle flux unit; $1 \mathrm{pfu}=1$ proton per $\left(\mathrm{cm}^{2} \mathrm{~s} \mathrm{sr}\right)$ ] in the $>10 \mathrm{MeV}$ energy channel of the Geostationary Operational Environmental Satellite (GOES) detectors. To understand the radio loudness of the RL CMEs, we divide them into three subgroups based on the wavelength range of the associated type II radio bursts. We denote the presence of type II radio bursts by y and Y (for yes) in metric and DH wavelengths, respectively; similarly, absence is indicated by $\mathrm{n}$ and $\mathrm{N}$ (for no). There are two y events with data gap (D) in the DH domain and $6 \mathrm{Y}$ events with metric data gap. Thus $\mathrm{nN}$ events are RQ CMEs, while $\mathrm{yY}, \mathrm{nY}$, and $\mathrm{yN}$ are the three subgroups of RL (FW) CMEs. The 2 yD and 6 DY events are also RL CMEs, but we do not include them in the analysis for kinematic properties. The yY events constitute the largest subset with 165 CMEs (out of 268 RL FW CMEs or 62\%), followed by $\mathrm{nY}$ events (69 CMEs or $26 \%$ ) and yN events (26 CMEs or $10 \%$ ). Thus FW CMEs with purely metric type II burst association (the $\mathrm{yN}$ events) are relatively small in number.

Figure 2 compares the speed and width distributions of the RQ CMEs and the three subsets of RL (FW) CMEs. The average speed increases in the following order: $\mathrm{nN}$ $(1117 \mathrm{~km} / \mathrm{s}), \mathrm{yN}(1186 \mathrm{~km} / \mathrm{s}), \mathrm{nY}(1340 \mathrm{~km} / \mathrm{s})$, and $\mathrm{yY}$
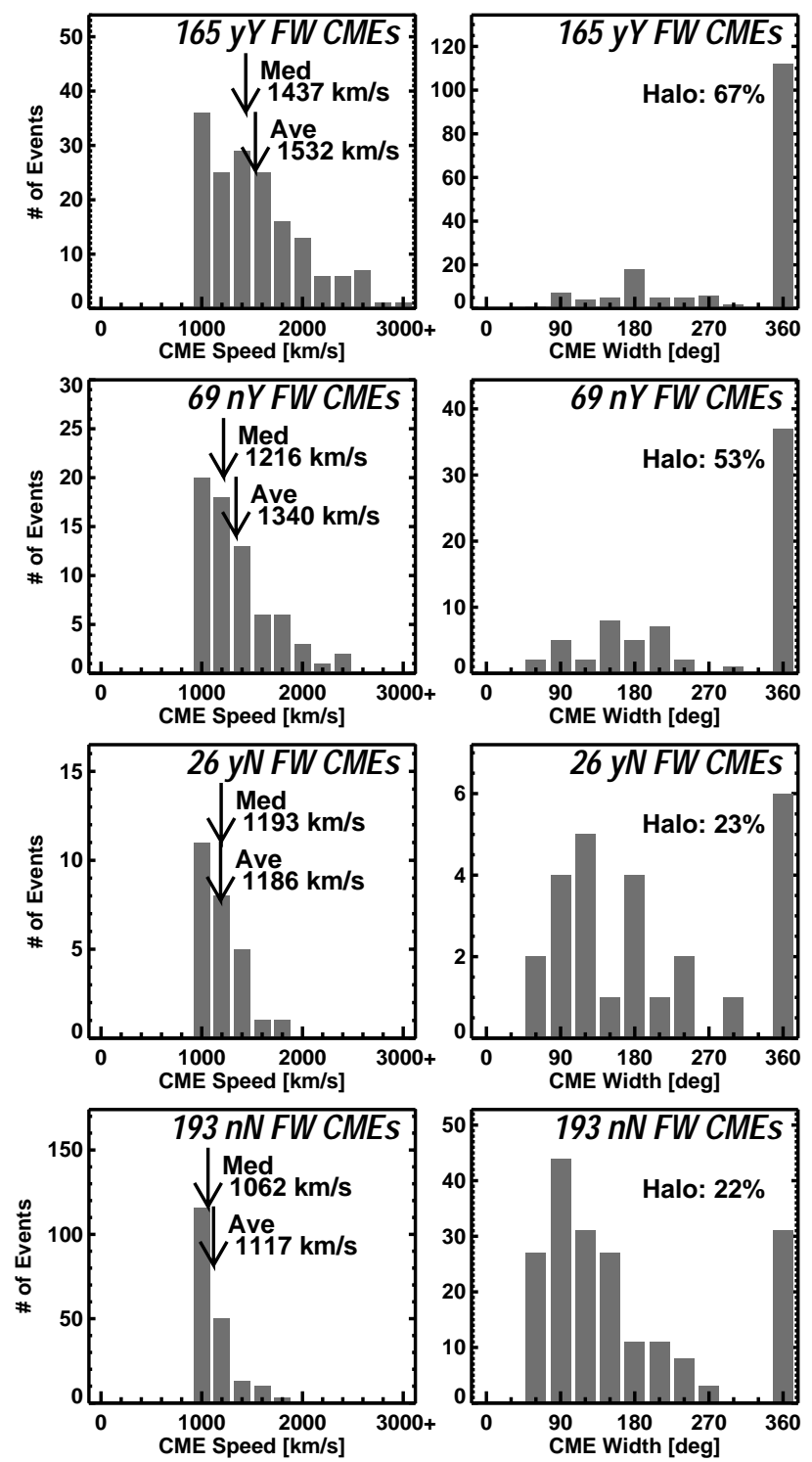

Fig. 2. Speed (left) and width (right) distributions of RQ (bottom row) and RL (3 upper rows) FW CMEs. Presence of type II radio bursts is indicated by y \& Y (for yes) in metric and DH wavelengths, respectively; similarly, absence is indicated by $\mathrm{n} \& \mathrm{~N}$ (for no). Thus $\mathrm{nN}$ events are RQ CMEs, while yY, nY, and yN represent RL CMEs. Note that 11 RL FW CMEs are not included because of data gap in one of the radio bands (metric or $\mathrm{DH}$ ). The speed bins have a size of $200 \mathrm{~km} / \mathrm{s}$, containing CMEs with speeds within $\pm 100 \mathrm{~km} / \mathrm{s}$ of the bin value. For example, the $1000 \mathrm{~km} / \mathrm{s}$ bin contains CMEs with speeds $\geq 900 \mathrm{~km} / \mathrm{s}$ but $<1100 \mathrm{~km} / \mathrm{s}$. For the width distribution, the bin size is $30^{\circ}$ and each bin contains CMEs within $\pm 15^{\circ}$ centered on the bin values.

$(1532 \mathrm{~km} / \mathrm{s})$. This is consistent with the hierarchical relationship between CMEs and type II radio bursts (Gopalswamy et al., 2005a). The yY events are similar to CMEs associated with the mkm type II bursts, although we did not check for the existence of the kilometric part. CMEs associated with 
Table 1. Properties of radio-quiet and radio-loud fast and wide (FW) CMEs ${ }^{\mathrm{a}}$.

\begin{tabular}{lcc}
\hline Property & Radio-quiet & Radio-loud \\
\hline Number of FW CMEs & $193(42 \%)$ & $268(58 \%)$ \\
Average speed & $1117 \mathrm{~km} / \mathrm{s}$ & $1438 \mathrm{~km} / \mathrm{s}$ \\
Average width & $86^{\circ}$ & $89^{\circ}$ \\
Fraction of halos & $16 \%$ & $60 \%$ \\
Median flare size & $\mathrm{C} 6.9$ & $\mathrm{M} 3.9$ \\
Fraction of backside CMEs & $55 \%$ & $25 \%$ \\
East-west asymmetry & -0.02 & 0.2 \\
Center-to-limb variation $_{\text {SEP association }}{ }^{\mathrm{a}}$ & increase & decrease \\
& none & $55 \%$ \\
\hline
\end{tabular}

${ }^{a}$ From Gopalswamy et al. (2008); ${ }^{b}$ based on the presence of SEP events with proton intensity $\geq 10$ pfu in the GOES $>10 \mathrm{MeV}$ energy channel.

purely metric type II bursts (yN events) are faster than the ones reported in Gopalswamy et al. (2005a) because of the cut off speed $(900 \mathrm{~km} / \mathrm{s})$ used here. The fraction of halos steadily increases in the same order as the speed: $\mathrm{nN}(22 \%)$, yN (23\%), nY (53\%), and y $(67 \%)$. Halo CMEs are thought to be wider on the average and wider CMEs are more massive so the fraction of halos in a population is indicative of the CME energy (Gopalswamy et al., 2007). Combining the speed and halo CME information we conclude that the RQ CMEs are the least energetic of the FW population (see also Table 1).

\subsection{CMEs with DH type II bursts}

When we look for CMEs associated with DH type II bursts without the criteria on speed and width, we identify more than 300 CMEs from 1996 to 2005. A list of these bursts along with the associated CMEs and their solar sources is available on line (http://cdaw.gsfc.nasa.gov/CME_list/radio/ waves_type2.html). CME information is not available for 10 events because of SOHO data gap (8 events) or uncertain CME association because of multiple CMEs (2 events). Each one of the remaining $334 \mathrm{DH}$ type II bursts is uniquely associated with a CME. Since these CMEs are radio loud owing to their association with DH type II bursts, we call them RL (DH) CMEs.

We have shown the speed and width distributions of all RL (DH) CMEs and the limb cases in Fig. 3. The limb CMEs originate far from the disk center (central meridian distances (CMD) in the range 60-90 ) and they are shown separately because they are not subject to projection effects. The speed distributions are approximate Gaussians with average speeds of $1228 \mathrm{~km} / \mathrm{s}$ (all) and $1398 \mathrm{~km} / \mathrm{s}$ (limb). The median speeds are not very different from the average speeds: $1151 \mathrm{~km} / \mathrm{s}$ (all) and $1393 \mathrm{~km} / \mathrm{s}$ (limb). Note that the average speed of all RL (DH) CMEs is slightly smaller than that of nY (1340)
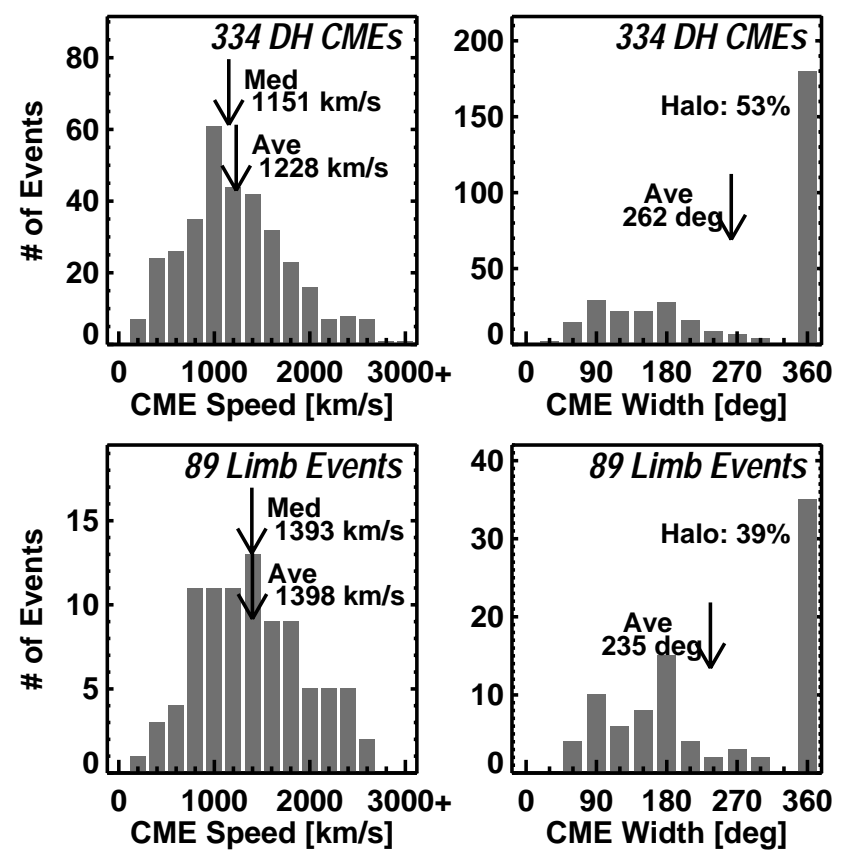

Fig. 3. Speed and width distributions of RL (DH) CMEs, i.e. CMEs associated with type II bursts in the DH wavelength domain. (top) All RL (DH) CMEs and (bottom) limb RL (DH) CMEs, i.e. originating in the central meridian distance range of $60-90^{\circ}$. The average (Ave) and median (Med) speeds are indicated on the plots. The bin sizes are as in Fig. 2.

and yY $(1532 \mathrm{~km} / \mathrm{s})$ events, which are higher-speed subsets of RL (DH) CMEs. The width distributions of RL (DH) CMEs in Fig. 3 show that they are generally wider and consist of a large fraction of halo CMEs. The average width of non-halo CMEs is well above $200^{\circ}$. The fraction of halos in the RL (DH) population (53\%) is very similar to that in the $\mathrm{nY}$ population. Another result worth pointing out is that RL (DH) CMEs with low speed are rare $(\sim 3 \%)$. The lowest bin $(200 \mathrm{~km} / \mathrm{s})$ in the limb CME distribution contains a single CME with a speed of $278 \mathrm{~km} / \mathrm{s}$. The CME identification is most likely incorrect, because there is another candidate with higher speed but was slowly accelerating. The $400 \mathrm{~km} / \mathrm{s}$ bin contains only three CMEs with speeds $358 \mathrm{~km} / \mathrm{s}, 373 \mathrm{~km} / \mathrm{s}$, and $381 \mathrm{~km} / \mathrm{s}$ with an average of $371 \mathrm{~km} / \mathrm{s}$.

\section{Type II association rate}

To make the association between CMEs and type II bursts more quantitative, we determine the type II association rate as a function of CME speed, width, and longitude (see Fig. 4). The type II association rate increases from $\sim 40 \%$ for the lowest bin $(1000 \mathrm{~km} / \mathrm{s}$, which includes CMEs from $900 \mathrm{~km} / \mathrm{s}$ to $1100 \mathrm{~km} / \mathrm{s}$ ) to $100 \%$ for the $2000 \mathrm{~km} / \mathrm{s}$ bin. The rate remains at $100 \%$ for CMEs faster than $2000 \mathrm{~km} / \mathrm{s}$. There was a single exception in the $2200 \mathrm{~km} / \mathrm{s}$ bin: the 7 April 

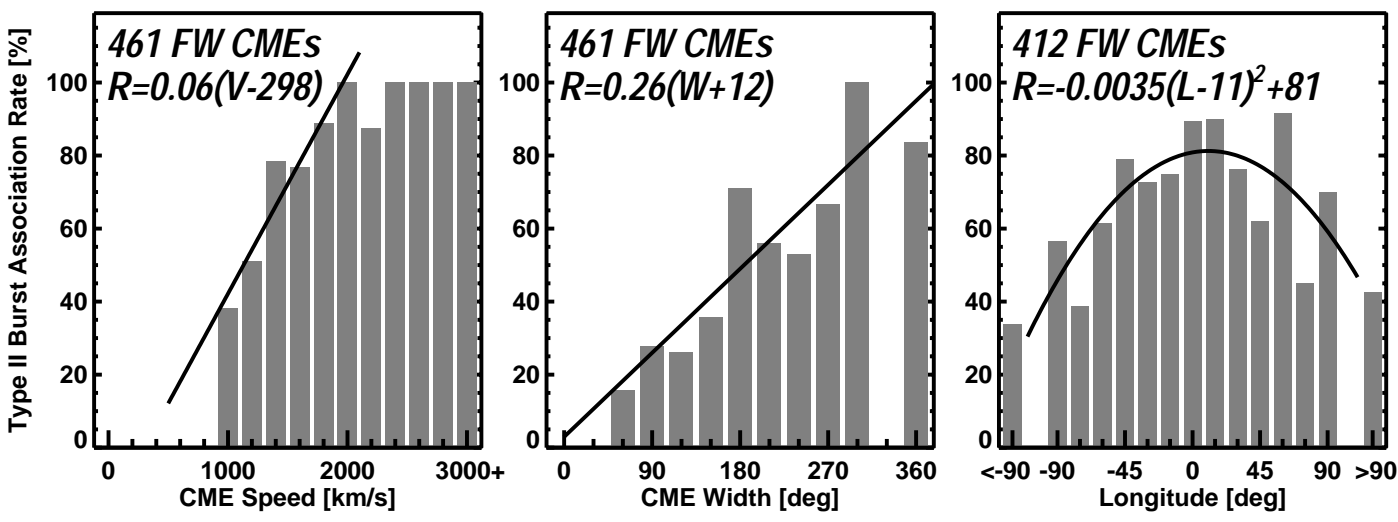

Fig. 4. Type II association rate of $461 \mathrm{FW}$ CMEs as a function of CME speed (V, left), width (W, middle) and source longitude (L, right). The equations of the straight-line fits are shown on the plots. In the longitude plot, 49 completely backside events have been excluded. The speed and width bin sizes are as in Fig. 2. The longitude bins have a size of $15^{\circ}$; each bin contains CMEs with longitudes within $\pm 7^{\circ} .5$ of the bin value.

$2001 \mathrm{CME}$ at 17:38 UT had a speed of $2198 \mathrm{~km} / \mathrm{s}$ but was radio-quiet. This CME will be examined in more detail at the end of this subsection. The association rate has a cutoff at $40 \%$ because we have restricted to FW CMEs. The linear increase of the type II burst association rate ( $\mathrm{R}$ in \%) with increasing CME speed (V in $\mathrm{km} / \mathrm{s})$ can be represented by the straight line, $\mathrm{R}=0.06$ (V-298). Figure 4 also shows that the type II association rate steadily increases with CME width, starting from $<20 \%$ for the $60^{\circ}$ bin to $100 \%$ for the $300^{\circ}$ bin. Note that only $\sim 85 \%$ of the full halos are associated with type II radio bursts, because some halos are slower than $900 \mathrm{~km} / \mathrm{s}$ and hence are not included here. The linear fit to the association rate is $\mathrm{R}=0.26(\mathrm{~W}+12)$, where $\mathrm{W}$ is the CME width in degrees. Finally, the association rate is higher $(\sim 80 \%)$ for CMEs originating near the disk center and falls off rapidly (by a factor of $\sim 2$ ) towards the limbs. The peak is also slightly shifted to the west, centered at $\sim \mathrm{W} 11$ as the fit shows: $\mathrm{R}=-0.0035(L-11)^{2}+81$, where $L$ is the source longitude in degrees. At least two possibilities exist for this center-to-limb behavior: (1) the limb CMEs are generally less energetic compared to the disk ones for a given speed because of projection effects, and (2) limb CMEs may have only a fraction of their shock surface visible to the observer, so their true association rate may be higher. This is especially true for metric type II bursts because they can be easily occulted. Cliver et al. (2004a) found that CMEs originating from up to $\sim 20^{\circ}$ behind the limb were associated with $\mathrm{DH}$ type II bursts. The plausible explanation for the predominance of western CMEs is the spiral magnetic field pattern upstream of the shock: for western CMEs, the accelerated electrons are channeled closer to the observer on the SunEarth line, while they are directed away from the observer for eastern CMEs.

Now we discuss the 7 April 2001 RQ CME whose skyplane speed is $2198 \mathrm{~km} / \mathrm{s}$ and the width is $152^{\circ}$. The two LASCO snapshots in Fig. 5 show the westward propagation of the CME. A EUV eruption can be seen just above the west limb in the 17:32 UT difference image as a narrow dark feature. The EUV images are from the Extreme-ultraviolet Imaging Telescope (EIT, Delaboudiniére et al., 1995) on board SOHO. This is clearly a behind-the-limb eruption. For CMEs originating within $\sim 30^{\circ}$ from limb, the measured skyplane speed is likely to be close to the true speed. However, the width of this CME seems to be heavily overestimated because of the peculiar position-angle distribution of the brightness. The CME consists of a narrow bright part with a width of only $\sim 80^{\circ}$ and an irregular (spiky) part mostly to the north. It is not clear if the northern spiky part belongs to this $\mathrm{CME}$ or not. If the true width is only $80^{\circ}$, the kinetic energy becomes considerably lower than a CME with the same speed but of higher width. Even with a width of $80^{\circ}$, the CME is still fast and wide, so the lack of type II radio emission may be due to other reasons to be discussed in Sect. 5 .

For comparison, Fig. 6 shows the association rate of DH type II bursts as a function of CME speed and width considering all CMEs between 1996 and 2005: fast and wide CMEs have a high degree of association with DH type II bursts. The association rate is around $10 \%$ for $1000 \mathrm{~km} / \mathrm{s}$ CMEs and climbs to $100 \%$ for $\geq 2000 \mathrm{~km} / \mathrm{s}$ CMEs. The $2200 \mathrm{~km} / \mathrm{s}$ bin has only $70 \%$ association rate because of three RQ CMEs out of the 10 CMEs in the speed range 2100 to $2299 \mathrm{~km} / \mathrm{s}$. The three RQ CMEs are: the 7 April 2001 CME described above (in Fig. 5), the narrow $\left(50^{\circ}\right.$ wide) $\mathrm{CME}$ on 31 October 2003 at 04:42 UT, and the 7 November $2003 \mathrm{CME}$ at 15:54 UT (occurred from behind the west limb). While the first two CMEs are clearly narrow, the radio-loudness of the last one is not clear due to intermittent gaps in the Wind/WAVES data. As for the type II burst association rate with CME width, we see that the maximum association rate is less than $47 \%$. Halo CMEs have the highest DH type II association rate. In fact, this plot justifies the consideration of FW CMEs as an important subset for producing interplanetary type II bursts (see Sect. 2.3). 


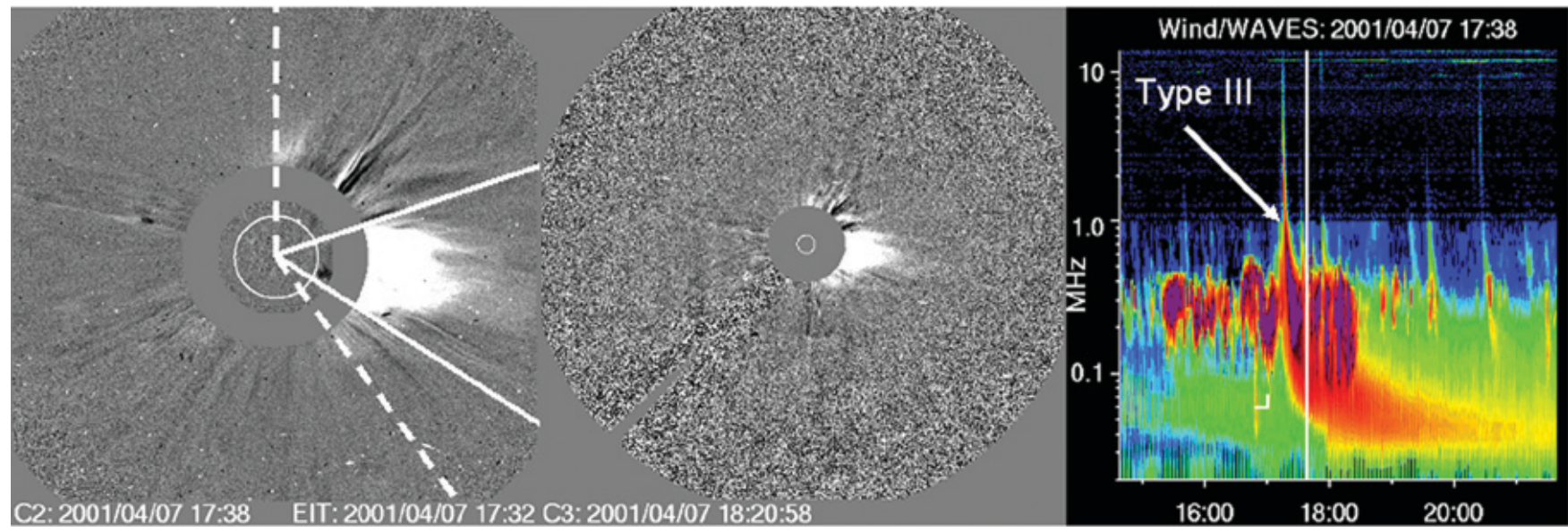

Fig. 5. SOHO/LASCO snapshots of the 7 April 2001 CME (the fastest RQ CME) at 17:38 UT (C2 image) and 18:20 UT (C3 image). The dashed lines (solid lines) show the CME edges of the overall disturbance (bright part) of the CME. The overall disturbance has a width of $152^{\circ}$, whereas the bright part has a width of only $80^{\circ}$. It is possible that the northern part of the CME is superposed with another weak CME. Wind/WAVES dynamic spectrum is shown on the right, with the type III burst indicated. There was no type II burst, so this CME is radio quiet.
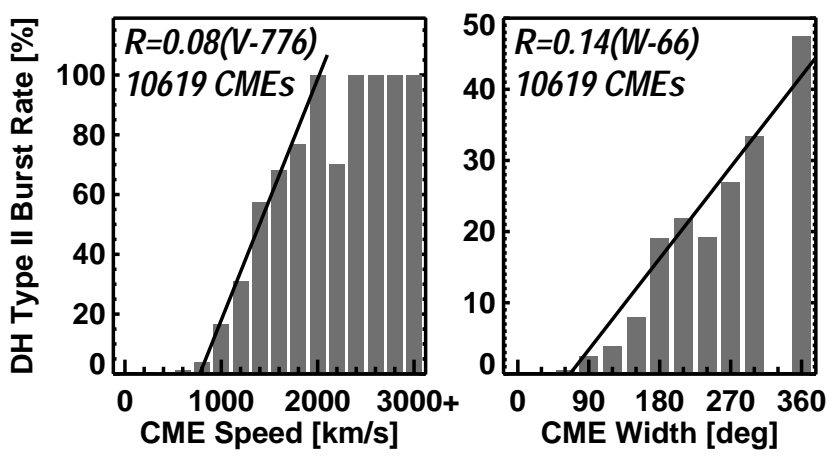

Fig. 6. Association rate (R) of DH type II burst as a function of CME speed $(\mathrm{V})$ and width $(\mathrm{W})$. The regression lines and their equations are shown on the plots. All the 10619 CMEs observed from 1996 to 2005 are considered. The rate is below $5 \%$ for $800 \mathrm{~km} / \mathrm{s}$ CMEs and reaches $100 \%$ for $2000 \mathrm{~km} / \mathrm{s}$ and faster CMEs. The rate is significant only for CMEs wider than $60^{\circ}$ and reaches a maximum of $47 \%$ for halo CMEs. The rate is less than $50 \%$ even for full halos because of backside events and slow halos. We have not plotted the rate variation with longitude because we do not have the heliographic coordinates for all the CMEs. The lower rate in the $2200 \mathrm{~km} / \mathrm{s}$ bin is discussed in the text.

\section{SEP association}

The lack of SEP events associated with RQ CMEs (see Table 1) is a significant result for space weather, because when one observes an FW CME at the Sun, it will not produce a large SEP event at Earth when it also lacks a type II burst. This does not mean all RL CMEs are SEPeffective. In fact, Table 1 shows that only slightly more than $50 \%$ of the RL FW CMEs have SEP association, which needs to be ex- plained. Furthermore, there are a few RQ CMEs that are associated with small SEP events ( $\mathrm{Ip}<10 \mathrm{pfu}$ ) (Gopalswamy et al., 2008). Thus we need to understand why some RQ CMEs are associated with SEP events (albeit small) and why some RL CMEs are not associated with SEP events.

\subsection{RQ CMEs with small SEP events}

The small SEP events (proton intensity $<10 \mathrm{pfu}$ ) associated with RQ CMEs can be used to constrain the characteristic speed of the ambient medium, provided the SEPs are due to CME-driven shocks (Reames, 1999). Some of the small SEP events may be impulsive events associated with the flare reconnection during the RQ CMEs. We show that some of these small SEP events are indeed gradual events, likely to be accelerated by CME-driven shocks. Table 2 lists the $14 \mathrm{RQ}$ CMEs accompanied by small SEP enhancements along with the following information: CME date, time (UT), central position angle (CPA), width ( $\mathrm{W}$ in degrees), speed ( $\mathrm{V}$ in $\mathrm{km} / \mathrm{s}$ ), location of eruption (heliographic coordinates), active region (AR) number, soft X-ray flare size, SEP intensity (Ip in pfu), and the approximate duration of the SEP event in days. The peak SEP intensities are all below 10 pfu. Events in which the peak intensity could not be determined accurately are denoted by a "?". Because of SEP events preceding or following some events, it is not possible to estimate the duration of some events; such events are denoted by $\mathrm{HiB}$ (having high background -2 events) or $\mathrm{F}$ (having following events -5 events). Among the 7 remaining SEP events, 5 have long ( $\geq 1$ day), 2 have short ( $<1$ day) durations. While the short duration ones may be impulsive SEP events, the longer duration ones are most likely gradual events attributable to shocks. 
Table 2. Properties of radio-quiet fast and wide CMEs with possible SEP association.

\begin{tabular}{cccccccccc}
\hline Date & UT & CPA $^{\mathrm{a}}$ & $\mathrm{W}^{\mathrm{a}}$ & $\mathrm{V}^{\mathrm{a}}$ & Location $^{\mathrm{b}}$ & AR\# & Flare & Ip $^{\mathrm{c}}$ & Dur (day) $^{\mathrm{d}}$ \\
\hline 28 Nov 1996 & $16: 50$ & 267 & 101 & 984 & N06W90 & 7997 & $\mathrm{C} 1.3$ & $<1$ & 2 \\
14 Nov 1997 & $10: 14$ & 79 & 86 & 1042 & N21E70 & 8108 & C4.6 & $1 ?$ & 1 \\
23 Apr 2000 & $12: 54$ & 360 & 360 & 1187 & W90b & - & DSF & 1 & 2 \\
12 Jul 2000 & $11: 06$ & 20 & 144 & 1124 & N17E27 & 9077 & X1.9 & $8 ?$ & F \\
27 Jul 2000 & $20: 06$ & 197 & 100 & 1119 & S12W77 & - & - & 10 & F \\
16 Sep 2000 & $13: 50$ & 198 & 100 & 1056 & Back & - & - & $?$ & HiB \\
17 Mar 2002 & $10: 34$ & 182 & 187 & 989 & S22E25 & 9871 & M1.3 & $8 ?$ & HiB \\
4 Jul 2002 & $20: 06$ & 270 & 168 & 957 & W90b & - & DSF & $<1$ & F \\
20 Aug 2002 & $08: 54$ & 237 & 122 & 1099 & S10W38 & 10069 & M3.4 & 2 & 1.5 \\
17 Mar 2003 & $19: 54$ & 291 & 96 & 1020 & S14W39 & 10314 & X1.5 & $<1$ & 0.3 \\
27 May 2003 & $22: 06$ & 215 & 123 & 1122 & S42W45 & - & PE & $<10 ?$ & F \\
22 Oct 2003 & $03: 54$ & 104 & 101 & 1163 & SE90b & 10486 & M3.7 & $<1$ & F \\
24 Oct 2003 & $02: 54$ & 113 & 123 & 1055 & S19E72 & 10486 & M7.6 & 2 & $>1$ \\
6 May 2005 & $11: 54$ & 277 & 129 & 1144 & S04W76 & 10756 & M1.3 & $<1$ & $<1$ \\
\hline
\end{tabular}

a The central position angle (CPA), and width (W) of CMEs are in degrees; the speed (V) is in km/s. ${ }^{b}$ The suffix " $b$ " indicates the behindthe-limb location of the eruption; e.g. SE90b means the eruption is behind the southeast limb. ${ }^{\mathrm{c}}$ Proton intensity in pfu (particle flux units); $1 \mathrm{pfu}=1$ particle per $\left(\mathrm{cm}^{2} \mathrm{~s} \mathrm{sr}\right)$. ${ }^{\mathrm{d}}$ Approximate duration of the SEP events in days. F denotes events followed by other events, and HiB denotes events occurring during elevated SEP background due to preceding events. In both cases, it is difficult to estimate the duration of the SEP event.

Let us look at the short-duration event on 17 March 2003 in more detail. The associated RQ CME has a speed of $1020 \mathrm{~km} / \mathrm{s}$ and a width of $96^{\circ}$. Figure 7 shows the SEP intensity as observed by SOHO's Energetic and Relativistic Nuclei and Electron (ERNE) experiment (Torsti et al., $1995)$ in five energy channels along with the GOES soft $\mathrm{X}$-ray light curve. The X1.5 flare associated with the SEP event was quite impulsive. The eruption occurred from AR 10314 (S14W39) well connected to SOHO. The SEP intensity peaked soon after the CME onset and lasted for only $\sim 8 \mathrm{~h}$. The short duration, weak intensity and well-connected location all suggest that this is an impulsive event caused by the X1.5 flare, rather than by the CME-driven shock. A preliminary analysis shows that the $\mathrm{He}^{3} / \mathrm{He}^{4}$ ratio is in the range 0.04 to 0.06 , two orders of magnitude higher than the nominal gradual SEP value $(0.0005)$. This ratio is consistent with an impulsive event, although we must caution that some gradual SEP events are also known to have enhanced $\mathrm{He}^{3} / \mathrm{He}^{4}$ ratio (see e.g. Torsti et al., 2003). The other shortduration event on 6 May 2005 was also associated with an impulsive flare from a well-connected location (S04W76). In addition to the 14 events in Table 2, there was another impulsive event detected by SOHO/ERNE, probably too weak to be detected by GOES. This event will be discussed in Sect. 5 .

Impulsive events are generally well connected, which is also true for the two events discussed above. The source locations in Table 2 show that all but one of the long-duration events are poorly connected (eastern or backside) events. For example, the 23 April 2000 long-duration SEP event is associated with a halo CME (average speed $1187 \mathrm{~km} / \mathrm{s}$ ) originat- ing from behind the west limb. The CME probably originates form AR 8951, which rotated behind the limb early on 21 April and hence is estimated to be $\sim 30^{\circ}$ behind the limb. Any associated flare must have been occulted. The particle intensity increases around the time the CME appeared in the LASCO field of view and continued for $\sim 2$ days, but the intensity never exceeded $1 \mathrm{pfu}$ (see Fig. 8). This is certainly a gradual event. Events like this point to the possibility of weak shocks driven by CMEs and accelerating low levels of SEPs. It must be pointed out that the source locations of the long-lasting SEP events imply that the associated CMEs are ejected at large angles to the Sun-Earth line, which is an important property of most of the RQ CMEs. This observational effect, noted as one of the reasons for the radio quietness, may also apply to the SEP events: the SEP events may not be so small for a well connected observer. Thus we conclude that some RQ CMEs (5 or possibly 12 out of 193) are definitely associated with gradual SEP events, suggesting that they drive weak shocks. Implications of such shocks will be discussed in Sect. 5 .

\subsection{RL CMEs and SEP events}

It is well known that almost all large SEP events are associated with DH type II radio bursts (Gopalswamy, 2003) and that the association rate increases when the radio bursts occur at metric and DH wavelengths (Cliver et al., 2004a) instead of just at metric type II bursts. Since type II bursts are indicative of SEP events, it is important to know what fraction of CMEs associated with type II bursts actually produce SEP events. This subsection describes how the subgroups of RL 
Table 3. SEP association rate among various FW CME populations.

\begin{tabular}{lcccccl}
\hline Type II & All & No SEP & HiB & $<1$ pfu & $\geq 1$ pfu & SEP Rate $^{\mathrm{c}}$ \\
\hline yY Events & 165 & 53 & 30 & 7 & 75 & $61 \%(56 \%)$ \\
nY Events & 69 & 25 & 17 & 5 & 22 & $52 \%(42 \%)$ \\
yN Events & 26 & 16 & 3 & 4 & 3 & $30 \%(13 \%)$ \\
nN Events $^{\mathrm{a}}$ & 193 & 144 & 35 & 5 & 9 & $9 \%(6 \%)$ \\
Radio DG $^{\mathrm{b}}$ & 19 & 8 & 9 & 0 & 2 & $20 \%(20 \%)$ \\
\hline
\end{tabular}

${ }^{\mathrm{a}} \mathrm{nN}$ events represent the RQ FW CMEs, of which the 14 SEP associated ones are listed in Table $2 .{ }^{\mathrm{b}}$ Includes 11 events with no radio data and 8 events with data gap (DG) either in the metric (6) or in the DH band (2). ${ }^{\mathrm{c}}$ Values in parentheses exclude $<1$ pfu events.
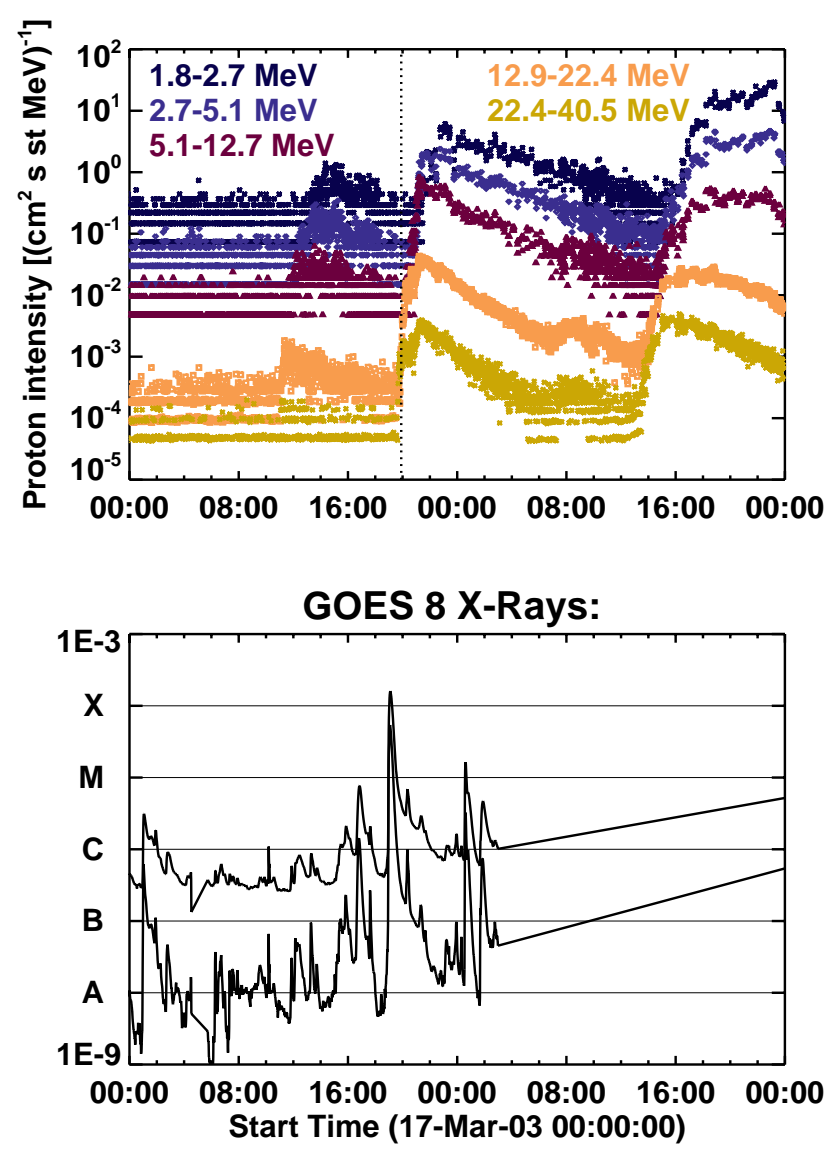

Fig. 7. (top) SEP intensity associated with the 17 March 2003 RQ CME from SOHO/ERNE in 5 energy channels. The CME onset (19:54 UT) is marked by the vertical dotted line. The SEP event was preceded and followed by other SEP events, but all are well separated. (bottom) GOES soft X-ray intensity (in two energy channels: 1-8 A upper, and 0.5-4 lower) as a function of time, showing the X1.5 flare associated with the CME.

FW CMEs are associated with SEP events. We also show how the association rate is distributed among CMEs associated with type II bursts in different wavelength domains.
Table 3 lists the SEP association rate for various FW CME populations grouped according to their type II burst association (yY, $\mathrm{nY}, \mathrm{yN}$, and $\mathrm{nN}$ in column 1; also includes radio data gap (DG)). Column 2 gives the total number of FW CMEs in each type II category. Columns 3-5 give the number of events with high SEP background (HiB), tiny SEP events (proton intensity Ip $<1 \mathrm{pfu}$ ), and SEP events with $\mathrm{Ip} \geq 1 \mathrm{pfu}$, respectively. Because of the elevated SEP background, the actual intensity cannot be determined for the $\mathrm{HiB}$ events. Note that I $\geq 1 \mathrm{pfu}$ events include both major $(\mathrm{Ip} \geq 10 \mathrm{pfu})$ and minor $(1 \mathrm{pfu} \leq \mathrm{Ip}<10 \mathrm{pfu})$ SEP events (Gopalswamy et al., 2002). The last column gives the SEP association rate, separately for all SEP events and those with $\mathrm{Ip} \geq 1 \mathrm{pfu}$ in parentheses. We see that $\mathrm{yY}$ events have the largest SEP association rate $(61 \%)$, closely followed by $\mathrm{nY}$ events $(52 \%)$. CMEs associated with purely metric type II bursts (yN events) have an SEP association rate of only $30 \%$. The progressive increase in SEP association as one goes from $\mathrm{yN}$ to $\mathrm{nY}$ to $\mathrm{yY}$ events confirms the conclusions of Cliver et al. (2004a).

The $\mathrm{nN}$ events have a 9\% SEP association rate (all SEP events are small, $\mathrm{Ip}<10 \mathrm{pfu}$ ). The $\mathrm{nN}$ events with small SEPs are in deed the RQ FW CMEs listed in Table 2. For 19 FW CMEs, there was data gap at radio wavelengths: 11 events with no radio data at all and 8 events with data gap in one of the bands (metric or DH). Two SEP events occurred during the data gaps. Note that RL FW CMEs involving DH type II bursts (yY and nY events combined) have a 58\% SEP association rate. If we include the $\mathrm{yN}$ events, the rate drops to $55 \%$. This analysis confirms the importance of DH type II bursts as indicators of SEP events.

Figure 9 further illustrates the importance of type II bursts by comparing the SEP association rate of FW CMEs with and without type II bursts. When all the FW CMEs (irrespective of the type II presence) are considered, the SEP association rate steeply increases from below $20 \%$ for $1000 \mathrm{~km} / \mathrm{s}$ CMEs to $100 \%$ for $\geq 2800 \mathrm{~km} / \mathrm{s}$ CMEs. The rate also increases with width, but less dramatically: $5 \%$ for $60^{\circ}$ wide CMEs to $40 \%$ for halo CMEs. With source longitude, the rate peaks around $\mathrm{W} 39$ with a maximum rate of $64 \%$. The rate remains at $20 \%$ for backside western events, but drops 

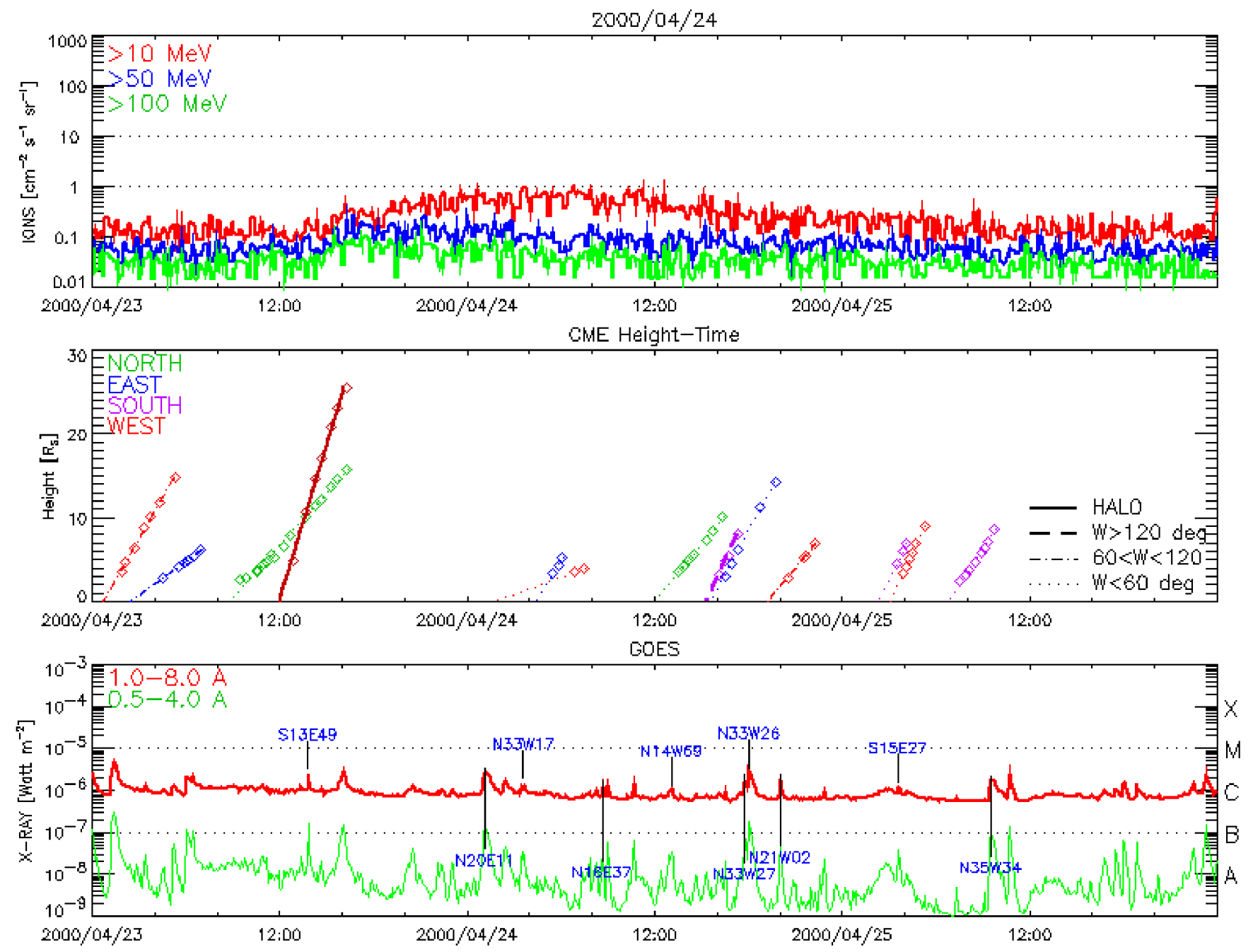

Fig. 8. (top) GOES SEP intensity in three channels (red: $>10 \mathrm{MeV}$, blue: $>50 \mathrm{MeV}$, and green: $>100 \mathrm{MeV}$ ) along with CME height-time plot and GOES soft X-ray plot for the 23 April 2000 RQ CME. (middle) CME height-time plots, the solid line indicating full halo CMEs, as noted. The CME of interest is the only halo in the time range plotted. (bottom) The flare locations (heliographic coordinates) are marked, but there is no flare location for the CME in question because it occurred behind the limb.

rapidly for eastern events, especially beyond E45. For the RL FW CMEs, the SEP association rate starts with a much higher value ( $\sim 40 \%$ ) for $1000 \mathrm{~km} / \mathrm{s}$ CMEs and reaches $100 \%$ for $\geq 2800 \mathrm{~km} / \mathrm{s}$ CMEs. With width, the rate increases from $\sim 25 \%$ ( $60^{\circ}$ wide) to reach a maximum of $65 \%$ for halos. The dependence of the rate with longitude has a similar behaviour, but has a higher peak rate ( $84 \%$ vs. $64 \%)$ and more westerly location (W48 vs. W39). For a given speed, width, and source longitude, the SEP association rate of FW CMEs is much higher if they are accompanied by type II bursts.

\subsection{RL (DH) CMEs and SEP events}

As we discussed in Sect. 2.3, the number of RL (DH) CMEs (334) is higher than that of the RL FW CMEs (268) because some $<900 \mathrm{~km} / \mathrm{s}$ CMEs produced DH type II bursts. Sixty- four of the RL (DH) CMEs had high background (HiB) levels of SEP intensity due to pervious eruptions, so we exclude them from the analysis. Of the remaining 270 events, 143 (or $53 \%$ ) had definitely no SEP events. The remaining $127 \mathrm{RL}$ (DH) CMEs (or 46\%) had definite SEP association, including 16 events with SEP Ip $<1$ pfu. Note that many of the HiB events also are real SEP events, but their intensity cannot be determined. The result that only about half of the DH type II bursts had SEP association is similar to what we found for RL FW CMEs, although the speed criterion and wavelength range of type II bursts are different.

Figure 10 illustrates the dependence of SEP association rate with the speed, width and source longitudes of RL (DH) CMEs. The SEP association rate is $<10 \%$ for CMEs slower than $600 \mathrm{~km} / \mathrm{s}$, but steadily increases to $100 \%$ for $2800 \mathrm{~km} / \mathrm{s}$ CMEs (similar to the rate for RL FW CMEs). The first width 

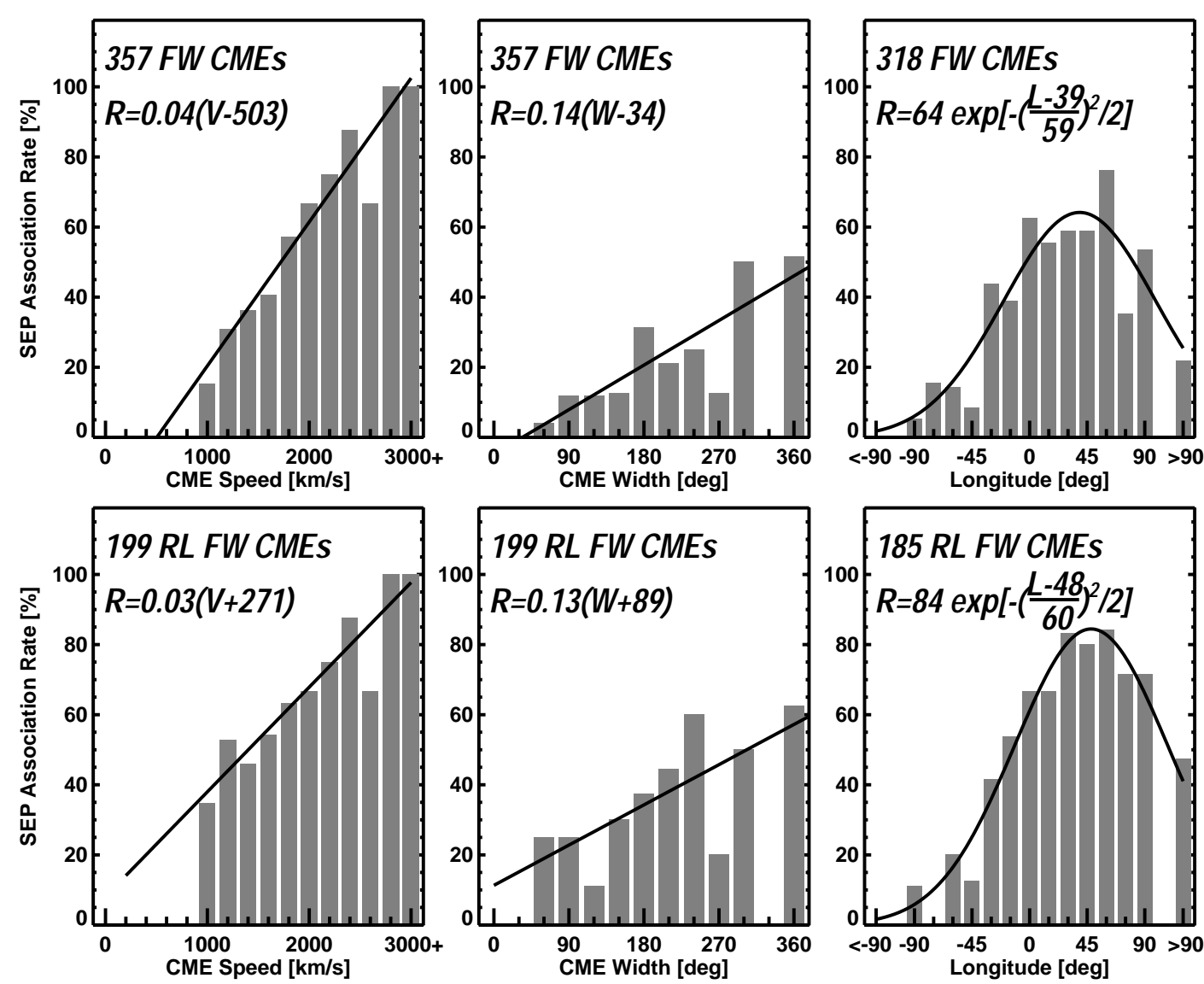

Fig. 9. SEP association rate of FW CMEs (top) and FW CMEs with type II bursts (RL FW CMEs, bottom) as a function of CME speed (V), width (W), and source longitude (L). All SEP events with intensity Ip $\geq 1$ pfu are included. Fitted curves to the distributions are shown on each plot.
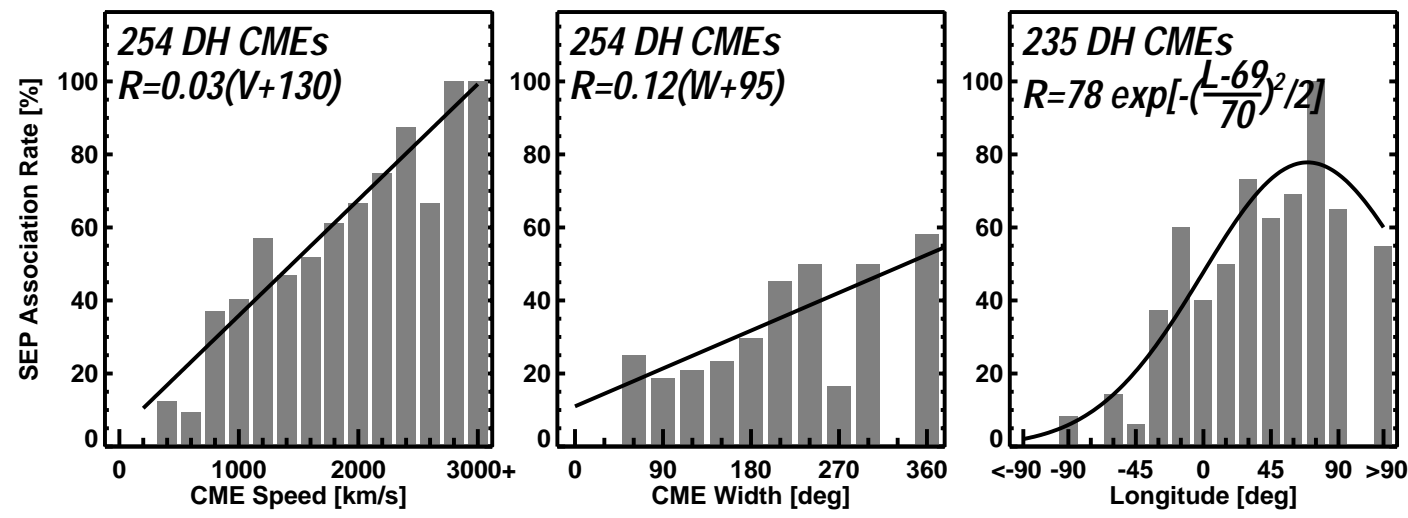

Fig. 10. SEP association rate $(\mathrm{R})$ as a function of CME speed (V km/s), width (W degrees) and source longitude (L degrees) for CMEs associated with DH type II bursts. The curves fitted to the rate are shown on the plots. Note that out of the 334 RL (DH) CMEs, only the 254 CMEs with SEP intensity $\geq 1$ pfu are included. The number of CMEs in the longitude distribution is smaller because source location is not available for some events.

bin with non-zero SEP association rate is $60^{\circ}$ (not due to selection). The SEP association rate increases from $\sim 20 \%$ for the $60^{\circ}$ wide CMEs to a maximum of $\sim 60 \%$ for full halos.
With the source longitude, the rate maximizes (78\%) at W63 and falls off on either side. The rate is very small for eastern longitudes especially for source locations east of E45 as in 

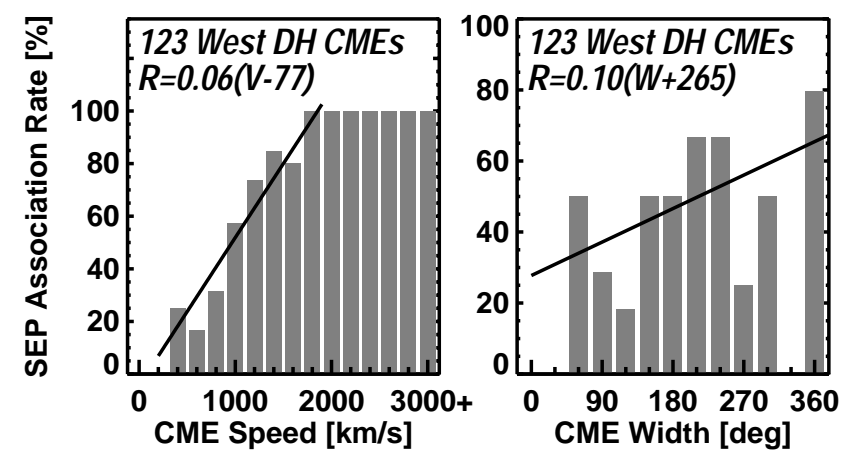

Fig. 11. Same as Fig. 10, but the SEP association rate for the western hemispheric CMEs accompanied by DH type II bursts.

Fig. 9. The dependence of SEP association rate with CME longitude is drastically different from that of DH type II bursts shown in Fig. 4: the SEP associated CMEs originate mostly from the western hemisphere, while the CMEs associated with DH type II bursts originate from both hemispheres. This readily explains why only about half of the CMEs associated with type II bursts are also associated with SEP events (see previous paragraph). The SEP association rate of western RL (DH) CMEs increases from $\sim 30 \%$ for $800 \mathrm{~km} / \mathrm{s}$ CMEs to reach $100 \%$ for $\geq 1800 \mathrm{~km} / \mathrm{s}$ CMEs (see Fig. 11). The rate for western halos is $80 \%$, much higher than the $\sim 60 \%$ for RL FW CMEs (Fig. 9). The SEP association rate of western CMEs accompanied by DH type II bursts is very similar to the DH type II burst association rate of all FW CMEs shown in Fig. 4.

The importance of western hemispheric origin for SEP producing CMEs is further illustrated in Fig. 12 in terms of the heliographic coordinates of all RL (DH) CMEs with and without SEP events (considering all SEP events with intensities $\geq 1 \mathrm{pfu}$ ). Note that CMEs associated with DH type II bursts occur at all longitudes, but those producing SEP events are predominantly in the western hemisphere because of the magnetic connectivity required between the observer near Earth and the SEP source region. The preferred western locations for SEP events is known for a long time (see e.g. McCracken, 1962). Thus, if a CME originating from the western hemisphere is accompanied by a DH type II burst, there is a high probability that it will produce an SEP event. This is a significant result for space weather applications.

\section{Influence of the ambient medium}

In the preceding subsections, it was found that CMEs with SEPs predominantly originated from the western hemisphere and those accompanied by type II bursts also had a slight western hemispheric bias. It was also reported earlier that the RQ CMEs originated at larger central meridian distances than the RL CMEs (see Table 1). It may be instructive to consider those RQ CMEs that bucked these trends. We need

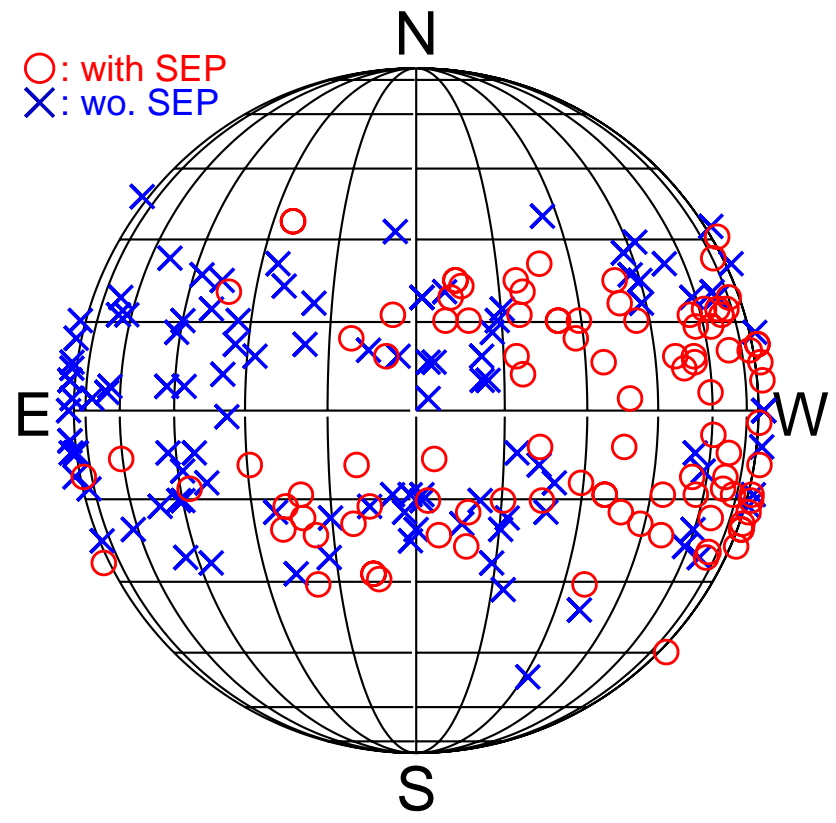

Fig. 12. Heliographic coordinates of CMEs accompanied by DH type II bursts. The circles and crosses distinguish RL CMEs with and without (wo) accompanying SEP events. All SEP events with $\mathrm{Ip} \geq 1$ pfu are included.

to select western RQ CMEs close to the central meridian, so we eliminated (i) all eastern events, (ii) western events at longitudes $>$ W60, and (iii) events at $\mid$ latitude $\mid>30^{\circ}$. This resulted in just 8 RQ CMEs with source locations overlapping with those of the disk RL FW CMEs. In this section, we investigate these events in more detail to understand the cause of their radio quietness.

Table 4 lists the 8 RQ CMEs along with information on the active region (AR) location (heliographic coordinates) and NOAA number, associated flares (soft X-ray importance), and the SEP association ( $\mathrm{N}$ - no SEPs; if there is SEP event, the peak intensity is given in pfu). Six of the events have numbered active regions, one is associated with a disappearing solar filament (DSF) observed by SOHO/EIT, and the remaining one is from an unnumbered region. As in the general population of RQ CMEs, the C-class flares are in the majority (see Gopalswamy et al., 2008); one flare is of M-class and the other of X-class. Only two CMEs were halos. Finally the CME sources are also close to the disk center (longitudes between W24 and W47; latitudes between S17 and N07), so the true speeds of CMEs are likely to be higher than the skyplane speed. The circumstances of eruption in each of these events are discussed briefly in the following.

23 September 1999 CME: This CME is associated with a filament eruption near an extended coronal hole in the southwest quadrant. Although the solar source is well connected, there is no SEP event above the detection thresholds of SOHO/ERNE and GOES. There are many streamers 
Table 4. List of RQ CMEs with source locations overlapping with RL disk CMEs.

\begin{tabular}{ccccccccc}
\hline Date & UT & CPA $^{\mathrm{a}}$ & W $^{\mathrm{a}}$ & Speed $^{\mathrm{b}}$ & Location & AR & Flare $^{\mathrm{c}}$ & SEP $^{\mathrm{d}}$ \\
\hline 23 Sep 1999 & $15: 54$ & 262 & 77 & 1150 & S14W47 & - & EIT/DSF & $\mathrm{N}$ \\
9 Feb 2000 & $19: 54$ & 360 & 360 & 910 & S17W40 & 8853 & C7.4 & $\mathrm{N}$ \\
20 Aug 2002 & $08: 54$ & 237 & 122 & 1099 & S10W38 & 10069 & M3.4 & 2 \\
17 Sep 2002 & $08: 06$ & 210 & 249 & 960 & S12W33 & 10114 & C8.6 & $\mathrm{N}$ \\
17 Mar 2003 & $19: 54$ & 291 & 96 & 1020 & S14W39 & 10314 & X1.5 & $<1$ \\
13 Apr 2003 & $09: 06$ & 301 & 91 & 936 & N07W49 & 10330 & C2.7 & $\mathrm{N}$ \\
9 Apr 2004 & $20: 30$ & 227 & 273 & 977 & S16W29 & 10588 & C2.8 & $\mathrm{N}$ \\
17 Feb 2005 & $00: 06$ & 360 & 360 & 1135 & S03W24 & - & C8.0 & N? \\
\hline
\end{tabular}

${ }^{a}$ The central position angle (CPA) and width (W) of CMEs are in degrees. ${ }^{b}$ The CME speed is in $\mathrm{km} / \mathrm{s}^{\mathrm{c}}{ }^{\mathrm{T}}$ The soft X-ray flare importance as listed in the online Solar Geophysical Data. ${ }^{d}$ Absence of SEP event is denoted by N (for no) and the presence is denoted by a number, which is the peak intensity of the SEP event in pfu. ${ }^{\mathrm{e}}$ A tiny SEP event was detected by SOHO/ERNE.

visible around the Sun, but none is affected by the eruption. Presence of the coronal hole is an indication that the Alfvén speed is high in the vicinity of the eruption region.

9 February 2000 CME: This is a halo CME originating from the southwest quadrant, in the vicinity of dimmer corona to the northeast and southwest as seen in EUV images. The eruption does not disturb the streamers in the vicinity. There is no SEP event in the ERNE or GOES data. A minor SEP increase observed $\sim 12 \mathrm{~h}$ after the CME onset is due to another RL CME on the next day.

20 August 2002 CME: This CME originates from an active region with a small coronal hole to the south of it. The associated flare is of $\mathrm{M}$ class. There is enhanced SEP background due to preceding CMEs but a new minor SEP enhancement (Ip 2 pfu) is observed in association with this CME. Wind/WAVES data shows a weak noise storm persistent during this event.

17 September 2002 CME: The background corona appears tenuous in this case. The CME is generally dimmer than the streamers seen in the coronagraphic images. The eruption slightly deflects the streamers above the west limb. There is no SEP enhancement in GOES or ERNE data. There is an ongoing noise storm at frequencies below $6 \mathrm{MHz}$.

17 March 2003 CME: This CME is associated with an X1.5 flare and a minor SEP event in both GOES and ERNE (see Fig. 7). The duration of the SEP event is $<8 \mathrm{~h}$, so the event is likely to be an impulsive event as discussed in Sect. 4.1. The CME width is probably overestimated. The CME shows a slight non-radial motion in the northwest direction, because the active region is located in the southwest quadrant. This may be due to the presence of a large coronal hole to the west and south of the active region. Also, the streamers in the west are unaffected by the CME.

13 April 2003 CME: This CME is much narrower than listed in the catalog. The coronal hole located to the west and south of the active region is a return of the coronal hole noted in connection with the 17 March 2003 event. No SEP event is found in the GOES or ERNE data.

9 April 2004: This CME starts out narrow but becomes large within the LASCO FOV. The CME is ejected into a tenuous region. The eruption is associated with a large group of intense type III bursts, but no SEP event is found in the GOES or ERNE data.

17 February 2005: This is the second of the two halos events in Table 4. The C8.0 flare is quite impulsive. A compact coronal hole is located to the north of the region. No SEP event is evident in the GOES data, but a tiny SEP event is detected by ERNE, likely to be an impulsive event.

The brief description of each of the event notes the presence of a tenuous environment into which the CMEs are ejected. This picture is necessarily qualitative, because we have not performed a similar analysis for the RL events. Nevertheless, this will be useful in understanding the role played by the ambient medium in deciding the ability of a CME to drive shocks and hence to accelerate electrons and protons (Gopalswamy et al., 2003b; Cliver et al., 2004b). The opposite extreme of the tenuous ambient medium is a medium with higher density such as streamers (Gopalswamy et al., 2004; Kahler and Vourlidas, 2005), preceding CMEs (Gopalswamy et al. 2004; Kahler 2001), which increase the efficiency of particle acceleration. Here we consider just the implications of the tenuous corona into which a $>900 \mathrm{~km} / \mathrm{s}$ CME is launched.

The average speed of the 8 disk RQ CMEs in Table 4 is $1023 \mathrm{~km} / \mathrm{s}$, which is even lower than the average speed of all RQ CMEs. However, when we apply projection correction, the true speed will be higher. For a crude estimate, we simply multiply the sky-plane speed by a factor of 1.6, which was obtained by comparing the average speeds of disk and limb halo CMEs (see Gopalswamy et al., 2007): the average deprojected speed of the 8 RQ CMEs thus becomes $\sim 1637 \mathrm{~km} / \mathrm{s}$. Of course, if we do the same thing to the disk RL CMEs, their space speed is likely to be even 
higher. Nevertheless, the fact remains that there are several RQ CMEs with speeds exceeding $1600 \mathrm{~km} / \mathrm{s}$. Only three of the 8 RQ CMEs had very small SEP enhancements. So, the question is why these CMEs are radio quiet and why they did not produce any large SEP event? Several explanations are possible.

(1) The simplest explanation is that these CMEs do not drive shocks. CMEs do not drive shocks when the characteristic speed (fast mode speed or Alfvén speed) in the ambient medium is higher than the CME speed. This means the Alfvén speed at coronal heights where metric and DH type II bursts are observed (1.5-3 $R_{E}$ from the Sun center) is $\sim 1600 \mathrm{~km} / \mathrm{s}$. Much higher Alfvén speeds are known to exist in coronal holes, while the streamers have much lower Alfvén speeds (see e.g. Esser et al., 1999). It is expected that the quiet coronal regions have intermediate values. When the corona is tenuous, the Alfvén speed can significantly increase. The relative change in the Alfvén speed (Va) depends on the relative changes in the coronal density (n) and magnetic field (B) according to $\mathrm{dVa} / \mathrm{Va}=\mathrm{dB} / \mathrm{B}-0.5 \mathrm{dn} / \mathrm{n}$, so for no change in magnetic field $(\mathrm{dB} / \mathrm{B} \sim 0)$, the Alfvén speed can go up by a factor of 2 when the density decreases by a factor of $4(\mathrm{dn} / \mathrm{n} \sim-4)$. Extended dark regions can be often seen in EIT images, which are not as dark as coronal holes, but darker than quiet regions. Fast and wide CMEs in such an ambient medium cannot drive shocks, so the type II bursts and SEP events will be absent. Kunches and Zwickl (1999) reported that SEP intensity is reduced when a coronal hole is present between the Sun-observer line and the solar source of the SEP event. This is consistent with the explanation above. However, this is not conclusive: Kahler (2004) did not find any bias against SEP production in fast solar wind regions (in which the Alfvén speed is expected to be higher), while Shen et al. (2006) did not find significant influence of the coronal holes on SEP intensity.

(2) The second possibility is that the RQ CMEs may be driving shocks, but the shocks are so weak that they do not accelerate enough electrons to produce detectable nonthermal emission. Weak shocks have low Alfvénic Mach numbers, which indicate higher Alfvén speed in the ambient medium. It is well known that shocks accelerate electrons and protons efficiently when the Mach number exceeds a critical value, which depends on the plasma beta (see Mann et al., 2003, for a discussion on this topic). For typical values of plasma beta in the outer corona ( $\sim 3 R_{E}$ from the Sun center), the critical Mach number is $\sim 1.5$. A $1600 \mathrm{~km} / \mathrm{s} \mathrm{CME} \mathrm{would}$ drive a subcritical shock if the Alfvén speed in the ambient medium is $\sim 1100 \mathrm{~km} / \mathrm{s}$. The small SEP events occasionally associated with RQ CMEs (e.g. the small SEP events in Tables 2 and 4) may be indicative of such weak shocks, provided they are not impulsive SEP events. We have shown in Sect. 4.1 that there are indeed some small gradual (i.e. shockrelated) SEP events associated with RQ CMEs.

(3) Finally, it must be pointed out that the generation of type II radio emission by the plasma emission process in- volves multiple steps: acceleration of electrons, production of Langmuir waves by the accelerated electrons, and the conversion of Langmuir waves into detectable electromagnetic radiation. Complete lack of large SEP events (at least in the well connected RQ CMEs) suggests that particle acceleration may be the main reason for radio quietness rather than the plasma emission process.

From the above discussion, we can conclude that the Alfvén speed in the ambient medium where plasma emission at metric and DH wavelengths originates can be as high as $1600 \mathrm{~km} / \mathrm{s}$ (when no shocks are formed) or $\sim 1100 \mathrm{~km} / \mathrm{s}$ (when weak shocks are formed). The lower extreme of the Alfvén speed can be estimated from the fact that some DH type II bursts are associated with CMEs of much lower speed $(<400 \mathrm{~km} / \mathrm{s})$. Recall from Sect. 2.3 that there are only 3 CMEs in the lowest bin $(400 \mathrm{~km} / \mathrm{s})$, which give a minimum average speed of $371 \mathrm{~km} / \mathrm{s}$. For an Alfvénic Mach number of 1.5 , the Alfvén speed is expected to be $\sim 247 \mathrm{~km} / \mathrm{s}$. When we consider metric type II bursts, the CME speed gets even lower ( $\sim 200 \mathrm{~km} / \mathrm{s}$, see Gopalswamy, 2006; Gopalswamy et al., 2005a), suggesting that the Alfvén speed could be below $200 \mathrm{~km} / \mathrm{s}$. It must be pointed out that the instances with very low coronal Alfvén speeds are very rare. We conclude that the coronal Alfvén speed can vary from $<200 \mathrm{~km} / \mathrm{s}$ to $1600 \mathrm{~km} / \mathrm{s}$ from event to event. This is in addition to the radial variation discussed by Mann et al. (1999), Gopalswamy et al. (2001a), and Mann et al. (2003). Consideration of such variation helps us understand not only RQ and RL CMEs, but also the relation between emission components produced at different radial distances from the Sun.

\section{Summary and conclusions}

This work concerns the ability of CMEs to produce type II radio bursts and SEPs, through the common process of particle acceleration by fast mode MHD shocks. We are not concerned about particle acceleration by flare processes that result in other types of radio emission such as type III bursts and impulsive SEP events. Using the extensive and uniform data on CMEs, SEPs, and type II radio bursts that became available during the $\mathrm{SOHO}$ era, we discuss how the CME properties such as speed, width and solar source longitude decide whether CMEs are associated with type II radio bursts and SEP events. We find that some radio-quiet CMEs are associated with small SEP events suggesting that these CMEs may drive weak shocks. We also find that some radio-loud CMEs are not associated with SEP events, mainly because of poor connectivity. Overall, we arrive at conclusions similar to Sheeley et al. (1984): either some fast and wide CMEs do not drive shocks or they drive weak shocks that do not produce detectable radio emission. The difference is we extend the analysis to the interplanetary type II bursts and solar energetic particle events, so we consider the general issue of particle acceleration, not just metric type II burst association. 
In addition, we arrive at the range of values for the Alfvén speed in the corona and near-Sun interplanetary medium.

We divide the fast and wide radio-loud CME population into three subgroups based on the wavelength range of type II bursts: (i) metric domain only, (ii) decametre-hectometric (DH) and longer wavelengths only, and (iii) all wavelengths (metric + DH and longer) and compared their properties. We find that the speed and fraction of halos in the three CME populations increase in the order (i), (ii), and (iii). The radioquiet CMEs are slower than all three radio-loud populations and have a lower fraction of halo CMEs as well. The SEP association rate also increases in the same order, pointing to the importance of longer wavelength type II bursts as indicators of SEP events.

We calculate the type II (metric and or DH) burst association rate as a function of CME speed, width and solar-source location. We find a steady increase in the association rate as the speed and width increase. The rate reaches $100 \%$ for large speeds $(\geq 2000 \mathrm{~km} / \mathrm{s})$, while the rate reaches $100 \%$ only for the widest non-halos. For full halos, the association rate is only $\sim 85 \%$ because some full halos are slow. The type II association rate peaks for CME source regions close to the disk center, with a slight western bias. When we consider the association rate of DH type II bursts (irrespective of the existence of a metric component) in the general CME population, we find that the association rate starts increasing rapidly around $1000 \mathrm{~km} / \mathrm{s}$ and reaches $100 \%$ at a speed of $2000 \mathrm{~km} / \mathrm{s}$. The rate also increases with width starting from about $60^{\circ}$ and reaching a maximum of $\sim 47 \%$ for full halos. Thus the definition of fast and wide CMEs with speeds $\geq 900 \mathrm{~km} / \mathrm{s}$ and widths $\geq 60^{\circ}$ is well founded.

We also find that when a fast and wide CME lacks type II emission, it also lacks a large SEP event. This is definitely useful knowledge. However, this does not mean that all CMEs with type II bursts are accompanied by SEP events: only about half of the CMEs associated with DH type II bursts are also accompanied by large SEP events. This is consistent with the lack of hemispheric preference for RL CME sources and the preferred western source location for SEP-associated CMEs.

Although most of the RQ CMEs are ejected at large angles to the Sun-Earth line, a subset of them have source distribution overlapping with that of the RL CMEs and the sources are located to the west of the disk center. We estimate that the deprojected average speed of these CMEs is $\sim 1600 \mathrm{~km} / \mathrm{s}$. Lack of type II radio bursts implies that these CMEs ought to be propagating through the corona and interplanetary medium where the Alfvén speed is relatively high. No shock implies an Alfvén speed of at least $1600 \mathrm{~km} / \mathrm{s}$, whereas weak subcritical shock implies an Alfvén speed of at least $1100 \mathrm{~km} / \mathrm{s}$. This is much higher than the nominal $500 \mathrm{~km} / \mathrm{s}$ usually assumed for the corona. Occasionally, the Alfvén speed can be below $200 \mathrm{~km} / \mathrm{s}$ as inferred from the speed distribution of CMEs associated with type II radio bursts. These results illustrate the highly inhomogeneous nature of the corona and interplanetary medium causing the spatial and temporal variation of the Alfvén speed by a large factor.

Acknowledgements. This work was supported by NASA's LWS TR\&T and SR\&T programs. We thank the anonymous referees for their helpful suggestions, which helped improve the presentation of the paper.

Topical Editor R. Forsyth thanks two anonymous referees for their help in evaluating this paper.

\section{References}

Bougeret, J.-L., Kaiser, M. L., Kellogg, P. J., et al.: Waves: The Radio and Plasma Wave Investigation on the Wind Spacecraft, Space Sci. Rev., 71, 231-263, 1995.

Brueckner, G. E., Howard, R. A., Koomen, M. J., et al.: The Large Angle Spectroscopic Coronagraph (LASCO), Solar Phys., 162, 357-402, 1995.

Cliver, E. W., Kahler, S. W., and Reames, D. V.: Coronal shocks and solar energetic proton events, Astrophys. J., 605, 902-910, 2004a.

Cliver, E. W., Nitta, N. V., and Thompson, B. J.: Coronal Shocks of November 1997 Revisited: The CME Type II Timing Problem, Solar Phys., 225, 105-139, 2004b.

Delaboudiniére, J.-P., Artzner, G. E., Brunaud, J., et al.: EIT: Extreme-Ultraviolet Imaging Telescope for the SOHO Mission, Solar Phys., 162, 291-312, 1995.

Esser, R., Fineschi, S., Dobrzycka, D., Habbal, S. R., Edgar, R. J., Raymond, J. C., Kohl, J. L., and Guhathakurta, M.: Plasma Properties in Coronal Holes Derived from Measurements of Minor Ion Spectral Lines and Polarized White Light Intensity, Astrophys. J., 510, L63-L67, 1999.

Gopalswamy, N.: Solar and geospace connections of energetic particle events, Geophys. Res. Lett., 30, SEP 1-1, 8013, 2003.

Gopalswamy, N.: A Global Picture of CMEs in the Inner Heliosphere, in: The Sun the Heliosphere As an Integrated System, edited by: Poletto, G. and Suess, S. (New York: Springer), 201, 2004.

Gopalswamy, N.: Coronal Mass Ejections and Type II Radio Bursts, in: AGU Monograph 165, Solar eruptions and energetic particles, edited by: Gopalswamy, N., Mewaldt, R., and Torsti, J., p. 207, 2006.

Gopalswamy, N., Lara, A., Kaiser, M. L., and Bougeret, J.-L.: NearSun and near-Earth manifestations of solar eruptions, J. Geophys. Res., 106, 25 261-25 278, 2001a.

Gopalswamy, N., Yashiro, S., Kaiser, M. L., Howard, R. A., and Bougeret, J.-L.: Characteristics of coronal mass ejections associated with long-wavelength type II radio bursts, J. Geophys. Res., 106, 29219-29 230, 2001b.

Gopalswamy, N., Yashiro, S., Michałek, G., Kaiser, M. L., Howard, R. A., Reames, D. V., Leske, R., and von Rosenvinge, T.: Interacting Coronal Mass Ejections and Solar Energetic Particles, Astrophys. J., 572, L103-L107, 2002.

Gopalswamy, N., Yashiro, S., Lara, A., Kaiser, M. L., Thompson, B. J., Gallagher, P. T., and Howard, R. A.: Large solar energetic particle events of cycle 23: A global view, Geophys. Res. Lett., 30, SEP 3-1, 8015, 2003. 
Gopalswamy, N., Yashiro, S., Krucker, S., Stenborg, G., and Howard, R. A.: Intensity variation of large solar energetic particle events associated with coronal mass ejections, J. Geophys. Res., 109, A12105, doi:10.1029/2004JA010602, 2004.

Gopalswamy, N., Aguilar-Rodriguez, E., Yashiro, S., Nunes, S., Kaiser, M. L., and Howard, R. A.: Type II radio bursts and energetic solar eruptions, J. Geophys. Res., 110, A12S07, doi:10.1029/2005JA011158, 2005a.

Gopalswamy, N., Yashiro, S., Liu, Y., Michalek, G., Vourlidas, A., Kaiser, M. L., and Howard, R. A.: Coronal mass ejections and other extreme characteristics of the 2003 OctoberNovember solar eruptions, J. Geophys. Res., 110, A09S15, doi:10.1029/2004JA010958, 2005b.

Gopalswamy, N., Yashiro, S., and Akiyama, S.: Coronal mass ejections and space weather due to extreme events, Proceedings of the ILWS Workshop, Goa, India, 19-24 February 2006, edited by: Gopalswamy, N. and Bhattacharyya, A., p.79, 2006.

Gopalswamy, N., Yashiro, S., and Akiyama, S.: Geoeffectiveness of halo coronal mass ejections, J. Geophys. Res., 112, A06112, doi:10.1029/2006JA012149, 2007.

Gopalswamy, N., Yashiro, S., Xie, H., Akiyama, S.. AguilarRodriguez, E., Kaiser, M. L., Howard, R. A., and Bougeret, J. L.: Radio Quiet Fast and Wide Coronal Mass Ejections, Astrophys. J., 674, 560-571, 2008.

Kahler, S. W.: The correlation between solar energetic particle peak intensities and speeds of coronal mass ejections: Effects of ambient particle intensities and energy spectra, J. Geophys. Res., 106, 20 947-20 956, 2001.

Kahler, S. W.: Solar Fast-Wind Regions as Sources of Shock Energetic Particle Production, Astrophys. J., 603, 330-334, 2004.

Kahler, S. W. and Vourlidas, A.: Fast coronal mass ejection environments and the production of solar energetic particle events, J. Geophys. Res., 110, A12S01, doi:10.1029/2005JA011073, 2005.
Kunches, J. and Zwickl, R.: The effects of coronal hole on the propagation of solar energetic proton events, Radiat. Meas., 30, 281286, 1999.

Mann, G., Klassen, A., Estel, C., and Thompson, B. J.: Coronal Transient Waves and Coronal Shock Waves, ESA Special Publications 446, edited by: Vial, J.-C. and Kaldeich-Schümann, B., p.477, 1999.

Mann, G., Klassen, A., Aurass, H., and Classen, H.-T.: Formation and development of shock waves in the solar corona and the nearSun interplanetary space, A\&A, 329-336, 2003.

McCracken, K. G.: The cosmic-ray flare effect, 3, deductions regarding the interplanetary magnetic field, J. Geophys. Res., 67, 447-458, 1962.

Reames, D. V.: Particle acceleration at the Sun and in the heliosphere, Space Sci. Rev., 90, 413-491, 1999.

Sheeley Jr., N. R., Howard, R. A., Michels, D. J., Robinson, R. D., Koomen, M. J., and Stewart, R. T.: Associations between coronal mass ejections and metric type II bursts, Astrophys. J., 279, 839847, 1984.

Shen, C., Wang, Y., Ye, P., and Wang, S.: Is There Any Evident Effect of Coronal Holes on Gradual Solar Energetic Particle Events?, Astrophys. J., 639, 510-515, 2006.

Torsti, J., Valtonen, E., Lumme, M., et al.: Energetic Particle Experiment ERNE, Solar Phys., 162, 505-531, 1995.

Torsti, J., Laivola, J., and Kocharov, L.: Common overabundance of ${ }^{3} \mathrm{He}$ in high-energy solar particles, Astron. Astrophys., 408, L1-L4, 2003.

Yashiro, S., Gopalswamy, N., Michalek, G., St. Cyr, O. C., Plunkett, S. P., Rich, N. B., and Howard, R. A.: A catalog of white light coronal mass ejections observed by the SOHO spacecraft, J. Geophys. Res., 109, A07105, doi:10.1029/2003JA010282, 2004. 